\title{
List of Participants
}

Ablowitz Mark J.

Department of Applied Mathematics

University of Colorado at Boulder

Boulder, CO 80309-526 USA

过Email:markjab@boulder.colorado.edu 胥

\section{岁Aigner Andreas}

¿Department of Engineering Mathematics

University of Bristol

ỐUniversity Walk

DSS8 1TR Bristol, UK

Email: A.Aigner@bristol.ac.uk

Alagesan Thangavel

Department of Information and

Communications

Kwangju Inst. of Science and Technology

1, Oryong-dong, Puk-gu

500-712 Kwangju, Korea

Email: talagesan@hotmail.com

Alekseev George

Steklov Mathematical Institute

Russian Academy of Sciences

Gubkina str. 8,

1199911 Moscow, Russia

¿ Email: G.A.Alekseev@mi.ras.ru

\section{Andreassi Gabriele}

Dipartimento di Matematica

Õ Università degli Studi di Lecce

ণิ via Arnesano, 73100 Lecce

$\approx$ Italy

ते

กิ Barone Fiorella

อิDipartimento di Matematica

Università degli Studi di Bari

70100 Bari, Italy

Email:

\section{Beccaria Matteo}

Dipartimento di Fisi

Università degli Studi di Lecce via Arnesano, 73100 Lecce, Italy

Email:matteo.beccaria@le.infn.it

\section{Biondini Gino}

Department of Mathematics

Ohio State University

231 West 18th Avenue

43210 Columbus, Ohio, USA

Email: biondini@math.ohio-state.edu

\section{Blaszak Maciej}

Institute of Physics

A. Mickiewicz University

Umultowska 85

61-614 Poznam, Poland

Email: blaszakm@main.amu.edu.pl

\section{Boiti Marco}

Dipartimento di Fisica

Universit degli Studi di Lecce via Arnesano, 73100 Lecce, Italy

Email:Marco.Boiti@le.infn.it

\section{Boscolo Sonia}

Photonics Research Group

School of Engineering and Applied Sciences

Aston University

Aston Triangle

B4 7ET Birmingham, UK

Email:boscolsa@aston.ac.uk

\section{Bruschi Mario}

Dipartimento di Fisica

Università "La Sapienza"

P.le A. Moro 2

00185 Roma, Italy

Email:bruschi@roma1.infn.it

Cafarella Alessandro

Dottorato di Ricerca in Fisica

Dipartimento di Fisica

Università degli Studi di Lecce

via Arnesano, 73100 Lecce, Italy

Email:cafarella@le.infn.it 


\section{Capovilla Riccardo \\ Departamento de Fisica CINVESTAV-IPN}

Av. IPN 2508 - Col. C. Pedro Zacatenco

07300 Mexico City, Messico

Email:capo@fis.cinvestav.mx $\dot{0}$

\section{Chirilli Giovanni}

Dipartimento di Fisica

GUniversità degli Studi di Lecce

Yyia Arnesano, 73100 Lecce

क्षेtaly

$8 \stackrel{0}{\circ}$

Conte Robert

Commissariat a l'energie atomique

CEA-Saclay, F-91191 Gif-sur-Yvette

France

Email:Conte@drecam.saclay.cea.fr

Corianò Claudio

Dipartimento di Fisica

Dniversità degli Studi di Lecce

V. Via Arnesano, 73100 Lecce, Italy

Email:coriano@le.infn.it

GूNFN Firenze

\&̆tg. E. Fermi 2

\$0125 Firenze, Italy

Email:cuoco@fi.infn.it



Degiovanni Luca

Dipartimento di Matematica

Università di Torino

Via Carlo Alberto 10, 10100 Torino

Italy Email:degio@dm.unito.it กे

\section{De Lillo Silvana}

Dipartimento di Matematica e Informatica

Università di Perugia

Via Vanvitelli 1, 06123 Perugia

Italy

Email:Silvana.delillo@pg.infn.it

Delle Side Domenico

Corso di laurea in Fisica
Università degli Studi di Lecce via Arnesano, 73100 Lecce, Italy

De Matteis Giovanni

Scuola Normale Superiore

Piazza dei Cavalieri 7

56100 Pisa, Italy

Email:dematteis@sns.it

\section{D'Innocenzo Antonio}

Dipartimento di Fisica

Università degli Studi di Lecce

via Arnesano, 73100 Lecce, Italy

Email:dinnocenzo@le.infn.it

\section{Dobrowolski Tomasz}

Instytut Fizyki AP

Ul. Podochorazych 2, 30-084 Cracow, Poland

Email:sfdobrow@cyf-kr.edu.pl

Dubrovin Boris

Mathematical Physics Sector

SISSA

via Beirut 2-4

34013 Trieste, Italy

Email:dubrovin@sissa.it

\section{Enolski Victor}

University of Salerno

Salerno, Italy

Email:enolskii@sa.infn.it

\section{Estevez Pilar Garcia}

Area de Fisica teorica, Facultad de Ciencias

Universidad de Salamanca

37008 Salamanca, Spain

Email:pilar@sonia.usal.es

\section{Fedele Renato}

Dipartimento di Scienze Fisiche

Università Federico II

Monte S.Angelo - via Cinthia

80126 Napoli, Italy

Email:renato.fedele@na.infn.it 


\section{Flach Sergej}

Max-Planck-Inst. für

Physik Complexe Systeme

Nöthnitzer Str. 38

D-01187 Dresden, Germany

Email:Flach@mpipks-dresden.mpg.de

\section{$\frac{\dot{0}}{\mathscr{S}}$ Gandarias Maria Luz}

Departamento de Matematicas

Universidad de Cadiz

Ap. 4011510 Puerto Real (Cadiz)

ญpain

Email:marialuz.gandarias@uca.es

:

\section{Garola Claudio}

Dipartimento di Fisica

Università degli Studi di Lecce

via Arnesano, 73100 Lecce, Italy

Email:garola@le.infn.it

$\stackrel{\circ}{\circ}$

Gerdjikov Vladimir S.

Institute for Nuclear Research and Nuclear

Energy

Bulgarian Academy of Science

Tzarigradsko chaussee 72

1784 Sofia, Bulgaria

Email:gerjikov@inrne.bas.bg

\section{Grahovski Georgi}

Institute for Nuclear Research and Nuclear Energy

Tzarigradsko chaussee 72

BG-1784 Sofia, Bulgaria

ฮี Email:Grah@inrne.bas.bg

$\overline{3}$

\section{Grava Tamara}

तi Department of Mathematics

in Imperial College

ธิ 180 Queens Gate

SW7 2BZ London, UK

Email.t.grava@ic.ac.uk

\section{Grundland Michel}

Centre de Recherches Mathematiques

Universite de Montreal

C.P. 6128 Succ. Centre-Ville
Montreal (Qubec) H3C 3J7

Canada

Email:grundlan@crm.umontreal.ca

\section{Guzzi Marco}

Dipartimento di Fisica

Università degli Studi di Lecce via Arnesano, 73100 Lecce, Italy

Email:marco.guzzi@le.infn.it

\section{Habibullin Ismagil}

Ufa Scientific Center

Institute of Mathematics

112 Chernyshevski str.

450077 Ufa, Russia

Email:Ihabi@imat.rb.ru

\section{Hone Andrew}

Institute of Mathematics and Statistics

University of Kent

CT2 7NF Canterbury, UK

Email:anwh@ukc.ac.uk

\section{Horvathy Peter}

Lab. de Mathematiques et Physique

Théorique

Universite de Tours

Parc de Grandmont

F-37200 Tours, France

Email:Horvathy@univ-tours.fr

Jaworski Marek

Institute of Physics

Al. Lotnikow $32 / 46$

02-660 Warsawa, Poland

Email:jawor@ifpan.edu.pl

\section{Kodama Yuji}

Department of Mathematics

Ohio State University

231 West 18th Avenue

43210 Columbus, Ohio, USA

Email:kodama@math.ohio-state.edu

\section{Konno Kimiaki}

Department of Physics, Coll. of Sc. Tech. 
Nihon University

1-8 Kanda-Surugadai, Chiyoda-ky

Tokyo 101-8308, Japan

Email:konno@phys.cst.nihon-u.ac.jp

\section{Konopelchenko Boris}

Dipartimento di Fisica

Università di Lecce

via Arnesano, 73100 Lecce

Italy

Email:konopel@le.infn.it

\section{Konotop Vladimir}

Complexo Interdisciplinar

University of Lisbon

Av. Prof. Gama Pinto 2

1649-003 Lisbon, Portugal

Email:Konotop@cii.fc.ul.pt

\section{Kruskal Martin}

Department of Mathematics

Rutgers, The State University of NJ

Hill Center, 110 Frelinghuysen Road,

Piscataway, NJ 08854, USA

Email:kruskal@math.rutgers.edu

\section{Kyuldjiev Assen}

Institute for Nuclear Research and Nuclear

Energy

Tzarigradsko chaussee 72

1784 Sofia, Bulgaria

Email:kyuljiev@inrne.bas.bg

\section{Landolfi Giulio}

Dipartimento di Fisica

Università di Lecce

via Arnesano, 73100 Lecce

Italy

Email:giulio.landolfi@le.infn.it

\section{Lee Jyh-Hao}

Institute of Mathematics

Academia Sinica

11529 Taipei, Taiwan

Email:leejh@math.sinica.edu.tw

\section{Leo Mario}

Dipartimento di Fisica

Università di Lecce

via Arnesano, 73100 Lecce

Italy

Email:mario.Leo@le.infn.it

Leo Rosario Antonio

Dipartimento di Fisica

Università di Lecce

via Arnesano, 73100 Lecce

Italy

Email:rosario.leo@le.infn.it

\section{Leon Jerome}

Physique Mathematique et Theorique Universite de Montpellier

Place E. Bataillon

34095 Montpellier, France

Email:Leon@lpm.univ-montp2.fr

\section{Magnano Guido}

Dipartimento di Matematica.

Università di Torino

Via Carlo Alberto 10

10100 Torino, Italy

Email:Magnano@dm.unito.it

\section{Man'ko Margarita A.}

P.N. Lebedev Physical Institute

Leninskii Prospect 53

119991 Moscow, Russia

Email:mmanko@sci.lebedev.ru

\section{Marmo Giuseppe}

Dipartimento di Scienze Fisiche

Universit Federico II

Complesso Universitario di Monte

S. Angelo, Via Cinthia

80126 Napoli, Italy

Email:marmo@na.infn.it

Martina Luigi

Dipartimento di Fisica

Università di Lecce 
Via Arnesano 73100 Lecce

Italy

Email:martina@le.infn.it

\section{Maruta Akihiro}

Dep. of Electronics and Information

s. Systems

Graduate School of Engineering

Osaka University

2-1 Yamada-oka

565-0871 Suita, Osaka, Japan

ŏ Email:maruta@comm.eng.osaka-u.ac.jp

究

.

Centre for Nonlinear Studies

Landau Institute for Theoretical Physics

Ul. Kosygina 2

Morandi Giuseppe

Dipartimento di Fisica

Università di Bologna

Viale B. Pichat 6/2

40127 Bologna, Italy

Email:morandi@bo.infn.it

\section{Morfu Saverio}

$\approx$ Laboratoire Le2i

Universite de Bourgogne CNRS

BP 47870

21078 Dijon, France

Email:smorfu@u-bourgogne.fr

๙ิ

$\lesssim$ Moro Antonio

Dipartimento di Fisica

î Università di Lecce

ถิ Via Arnesano 73100 Lecce

Italy

Email:antonio.moro@le.infn.it

\section{Musette Micheline}

Dienst Theoretische Natuurkunde

Vrije Universiteit Brussel

Pleinlaan 2, B-1050 Brussels
Belgium

Email:mmusette@vub.ac.be

\section{Myrzakulov Ratbay}

Institute of Physics and Technology

National Academy of Sciences

of Kazakhstan

480082 Alma-Ata, Kazakhstan

Email:cnlpmyra@satsun.sci.kz

\section{Nieszporski Maciej}

Inst. Fizyki Teoretycznej

Uniwersytet w Bialimstoku

Ul. Lipowa 41

15-424 Bialystok, Poland

Email:maciejun@fuw.edu.pl

\section{Orlov Alexander}

P.P. Shirshov Institute of Oceanology

117851 Moscow, Russia

Email:orlovs@wave.sio.rssi.ru

\section{Paladini Fabio}

Dipartimento di Fisica

Università di Lecce

via Arnesano, 73100 Lecce

Italy

Email:Fabio.Paladini@le.infn.it

\section{Palese Marcella}

Dipartimento di Matematica

Università di Torino

Via Carlo Alberto 10

I-10123 Torino, Italy

Email:palese@.dm.unito.it

\section{Pashaev Oktay}

Department of Mathematics

Izmir Institute of Technology

Gulbahce Campus

35437 Urla-Izmir, Turkey

Email:pashaev@likya.iyte.edu.tr

\section{Pavlov Maxim}

Landau Institute for Theoretical Physics Kosygina 2 
7-095-1373244 Moscow, Russia

Email:maxim.pavlov@mtu-net.ru

\section{Pelloni Beatrice}

Department of Mathematics

University of Reading

RG6 6AX Reading, UK

Email:b.pelloni@rdg.ac.uk

is

\section{Pempinelli Flora}

Dipartimento di Fisica Đniversità degli Studi di Lecce yia Arnesano, 73100 Lecce

Htaly Email:Flora.Pempinelli@le.infn.it

Steklov Mathematical Institute

Subkin str. 8,

19991 Moscow, Russia

Email:pogreb@mi.ras.ru

Prinari Barbara

Ëniversità di Lecce

Fria Arnesano, 73100 Lecce

คิ̆taly

Email:barbara.prinari@le.infn.it

\section{$\widehat{D}$}

\section{Protogenov Alexander}

Institute of Applied Physics

乙. Tussian Academy of Sciences $\Phi_{46}$ Ul'yanov str.,

व్GSP-120 603950 Nizhny Novgorod

Russia

§Email:alprot@appl.sci-nnov.ru

ते

\section{iRagnisco Orlando}

Dipartimento di Física

Università di Roma TRE

Via Vasca Navale 84

00146 Roma, Italy

Email:ragnisco@fis.uniroma3.it

\section{Renna Luigi}

Dipartimento di Fisica
Università di Lecce

via Arneasano, 73100 Lecce

Italy

Email:renna@le.infn.it

\section{Sabatier Pierre}

Physique Mathematique

Universite de Montpellier II

Place E. Bataillon

34095 Montpellier Cx5

France

Email:Sabatier@LPM.univ-montp2.fr

\section{Salerno Mario}

Dipartimento di Scienze Fisiche

E.R. Caianiello

Università di Salerno

via S. Allende

I-84081 Baronissi (SA), Italy

Email:salerno@sa.infn.it

\section{Sanchini Giampaolo}

Dipartimento di Matematica e Informatica Università di Perugia

Via Vanvitelli 1, 06123 Perugia

Italy

Email:giampaolo.sanchini@pg.infn.it

\section{Santini Paolo Maria}

Dipartimento di Fisica

Università di Roma "La Sapienza"

P.le A. Moro 2

00185 Roma, Italy

Email:paolo.santini@roma1.infn.it

\section{Scolarici Giuseppe}

Dipartimento di Fisica

Università di Lecce

via Arnesano, 73100 Lecce

Italy

Email:scolarici@le.infn.it

\section{Skarka Vladimir}

CNRS Laboratoire POMA, UMR 6136

University of Angers

2, boulevard Lavoisier 
49000 Angers, France

Email:vladimir.skarka@univ-angers.fr

\section{Soliani Giulio}

Dipartimento di Fisica.

Università di Lecce

\&ia Arnesano, 73100 Lecce

taly

Email:giulio.soliani@le.infn.it

ठ

\section{Solombrino Luigi}

Đipartimento di Fisica

Università di Lecce

Kia Arnesano, 73100 Lecce

Straly

Email:luigi.solombrino@le.infn.it

\section{.}

Centro de Fisica da Materia Condensada ⿹्र niversidade de Lisboa

Av. Gama Pinto 2

1649-003 Lisboa, Portugal

Email:spire@cii.fc.ul.pt

\section{Tanaka Keiji}

T.

2-15 Ohara-Kamifukuoka-shi

政56-8502 Saitama, Japan

Email:kj-tanaka@kddi.com

\section{Tempesta Piergiulio}

Centre de Recherches Mathematiques

Õniversite de Montreal

Pavillon Andre-Aisenstadt, Case postale $\approx 6128$

ॠMontreal (Qubec) H3C 3J7

inCanada

Email:tempesta@crm.umontreal.ca

\section{Tomassini Paolo}

ILIL IPCF-CNR Area della Ricerca di Pisa

Via G. Moruzzi 1

56127 Pisa, Italy

Email: tomassini@ifam.pi.cnr.it

Tulczyjew Vlodzimierz $M$.

Dipartimento di Fisica

Università di Camerino

62032 Camerino, Italy

Email:tulczy@camserv.unicam.it

\section{Vanossi Andrea}

Dipartimento di Fisica

Università di Modena e Reggio Emilia

Via Campi 213/a, 41100 Modena

Italy

Email:vanossi.andrea@.unimo.it

\section{Winterroth Ekkehart}

Dipartimento di Matematica

Università di Torino

Via Carlo Alberto 10

I-10123 Torino, Italy

Email:ekkehart@.dm.unito.it

\section{Wu Derchyi}

Institute of Mathematics

Academia Sinica

Nankang 11529 Taipei, Taiwan

Email:mauudc@ccvax.sinica.edu.tw

\section{Zolotaryuk Yaroslav}

Section of Mathematical Physics IMM

Technical University of Denmark

Richard Petersen Plads

2800 Lyngby, Denmark

Email:yz@imm.dtu.dk 